\title{
Recherche et pratique musicales en bibliothèque : une expérience partagée entre bibliothécaires, élèves et professeurs
}

\section{Cécile Grand et Mathilde Vittu}

\section{(2) OpenEdition}

Journals

Édition électronique

URL : http://journals.openedition.org/rbnu/292

DOI : $10.4000 /$ rbnu. 292

ISSN : 2679-6104

Éditeur

Bibliothèque nationale et universitaire de Strasbourg

\section{Édition imprimée}

Date de publication : 23 mai 2018

Pagination : $42-49$

ISSN : 2109-2761

Référence électronique

Cécile Grand et Mathilde Vittu, «Recherche et pratique musicales en bibliothèque : une expérience partagée entre bibliothécaires, élèves et professeurs », La Revue de la BNU [En ligne], 17 | 2018, mis en ligne le 05 septembre 2018, consulté le 10 décembre 2020. URL : http://journals.openedition.org/ rbnu/292 ; DOI : https://doi.org/10.4000/rbnu.292

\section{(c) (i) (2)(2)}

La Revue de la BNU est mise à disposition selon les termes de la Licence Creative Commons Attribution - Pas d'Utilisation Commerciale - Partage dans les Mêmes Conditions 4.0 International. 


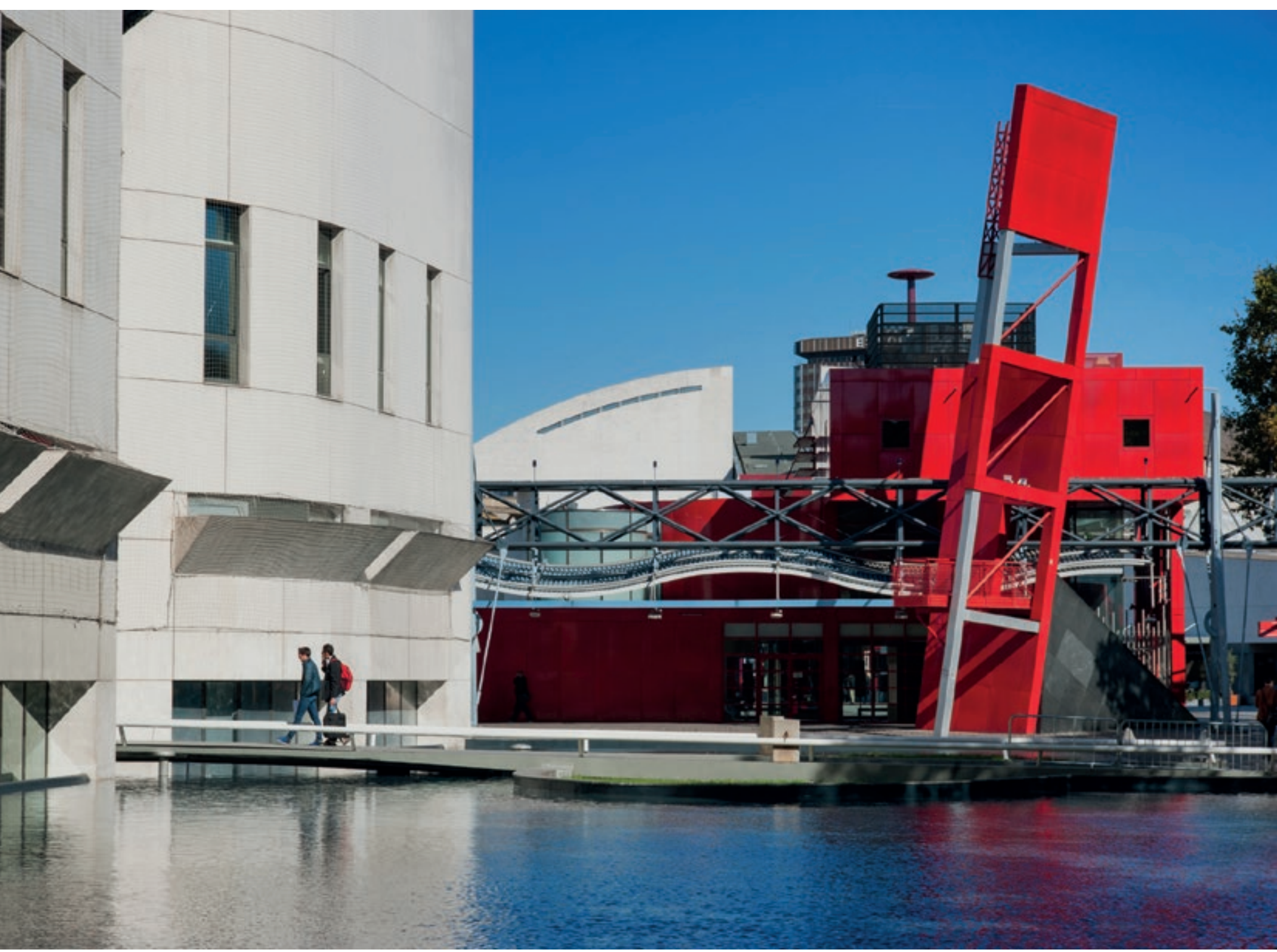

La Cité de la musique à Paris 


\title{
RECHERCHE ET PRATIQUE MUSICALES EN BIBLIOTHEQUE : une expérience partagée entre bibliothécaires, élèves et professeurs
}

\author{
PAR CÉCILE GRAND ET MATHILDE VITTU
}

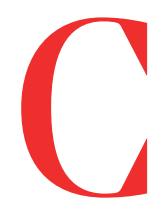

omme toutes les bibliothèques d'écoles spécialisées, celle du Conservatoire national supérieur de musique et de danse de Paris (CNSMDP) a toujours entretenu un lien très fort avec l'institution et ses pratiques pédagogiques. Ouverte à tous, son principal public est aujourd'hui celui des musiciens et dans une moindre mesure des danseurs élèves du Conservatoire. La manière dont ils fréquentent ou " contournent " le lieu est une source d'interrogation : malgré des moyens exceptionnels et une volonté politique historique de leur donner accès à ses ressources, la rencontre avec le lieu a parfois du mal à se faire, même si une (r)évolution se dessine.

\section{_ Une bibliothèque au cœur de l'enseignement}

La bibliothèque a été créée en 1795 , en même temps que le Conservatoire : l'article $\mathrm{X}$ du décret du 16 thermidor (3 août 1795) ${ }^{1}$ prévoyait déjà de doter l'institution d'« une Bibliothèque Nationale de Musique [...] composée d'une collection complète de partitions et d'ouvrages traitant de cet art ». Elle fut dès ses débuts très fortement liée à l'enseignement et à la pratique musicale : "Le règlement ne doit pas se borner à organiser les institutions indispensables à l'étude de la théorie générale de la musique ; il faut aussi qu'il fournisse à la pratique les moyens de transmettre les leçons utiles de l'expérience ; il faut que les ouvrages des maîtres de tous les temps et de toutes les nations, réunis dans la bibliothèque du Conservatoire, offrent aux recherches des jeunes artistes les conseils du savoir $[\ldots] »^{2}$.

Ses collections sont aujourd'hui riches de près de 300000 documents (partitions, livres, documents audiovisuels...), complétés par de nombreuses ressources numériques. Deuxième bibliothèque musicale publique de France, derrière le Département de la musique de la Bibliothèque nationale de France, elle est tout à la fois une bibliothèque patrimoniale qui rassemble des collections uniques et rares (manuscrits musicaux, imprimés anciens, archives...), une bibliothèque de référence pour la musique et la danse et une bibliothèque scolaire au service d'une communauté pédagogique spécialisée en musique et en danse.

Cette volonté politique d'établir un lien fort entre pratique artistique et étude théorique s'accompagne dès le départ de moyens adaptés, et la bibliothèque sera dotée tout au long de son histoire de moyens conséquents : des lieux aménagés, des collections uniques et des bibliothécaires qui entretiendront tous un lien fort avec l'école.

En 1801, les collections ${ }^{3}$ sont installées dans un bâtiment spécialement aménagé et dès 1806, il est possible de copier et de lire au piano des ouvrages $\mathrm{du}$ fonds dans des cabinets de musique adjacents. 
Les bibliothécaires seront tous musiciens jusqu'à une époque récente, certains comme Félicien David et surtout Hector Berlioz seront célèbres comme compositeurs. Leur lien très fort avec la musique, leur goût et leur culture seront à l'origine d'enrichissements conséquents, et très vite les collections de la bibliothèque du Conservatoire permettront aux élèves et professeurs d'accéder à des répertoires et à des esthétiques très variés.

Au début du $20^{\mathrm{e}}$ siècle, la bibliothèque du Conservatoire est une véritable «Bibliothèque nationale de musique " et met à disposition de son public, et notamment des élèves de l'institution, un outil unique en Europe - à tel point que devant la richesse et la valeur inestimable de ses collections (unica, éditions anciennes et manuscrits comme celui, autographe, du Don Giovanni de Mozart), il est décidé de la rattacher à la Bibliothèque nationale (1935). En 1942, les fonds anciens et précieux sont transférés dans cette dernière, et en 1964, la bibliothèque du Conservatoire est administrativement rattachée au Département de la musique.

Cette nouvelle situation administrative, qui lui permit de bénéficier de la compétence des conservateurs de la Bibliothèque nationale en matière de catalogage et de gestion des collections, eut cependant des conséquences sur sa place au sein de l'école. Les élèves ne purent plus accéder aussi facilement aux collections patrimoniales, et en abandonnant partiellement à la Bibliothèque nationale sa politique d'acquisition, la bibliothèque fut plus perçue comme une annexe de cette dernière que comme un lieu de proximité avec les sources musicales.

Cet état de fait sera exacerbé par l'émergence de la musicologie à l'université, avec en filigrane l'idée que la pratique appartient au Conservatoire, et la théorie à l'université.

\section{- Sur le site de la Cité de la musique : de nouveaux moyens, de nouvelles méthodes}

L'installation du Conservatoire dans un nouveau bâtiment en 1990 donna à l'institution l'opportunité de nuancer fortement cette vision et de renouer avec le dessein initial : une bibliothèque au cœur d'un enseignement musical et artistique large et ouvert.
À partir de cette date, des moyens très conséquents sont alloués à la Médiathèque Hector Berlioz ${ }^{4}$ pour développer un fonds de prêt à l'usage des élèves et des professeurs, pour enrichir les collections (musique, musicologie, histoire de la danse...), avec une attention particulière pour les répertoires contemporains.

Malgré cela, le lieu est relativement peu fréquenté et son public est essentiellement composé d'élèves des cursus de musicologie et d'écriture. La richesse musicale des collections offertes à la consultation $^{5}$ et les nombreuses ressources numériques accessibles à distance semblent ignorées des élèves : en dehors du prêt, la médiathèque peine à trouver son public.

En 2012, la direction de l'établissement a donc donné carte blanche au service pour mettre en place des actions qui permettraient aux élèves - et aux professeurs - de s'approprier l'outil dans son ensemble (collections physiques et ressources numériques).

Pour accompagner la médiathèque dans sa " reconquête » des lecteurs, la section de consultation a bénéficié de travaux de rénovation conséquents (430 $000 €)$ en 2014. Le cahier des charges était porté par l'une des bibliothécaires et privilégiait la qualité du lieu (espace, lumière, confort des installations, ergonomie de la banque d'accueil). L'un de ses points forts fut l'aménagement d'un espace ouvert, au sein même de la salle de lecture, pour accueillir des cours, pendant ou en dehors des heures d'ouverture au public.

Cette réorganisation des espaces autour de l'enseignement s'est accompagnée d'une réorganisation $\mathrm{du}$ mode de fonctionnement du service (heures d'ouverture, permanences, astreintes), de façon à élargir les heures d'accès au lieu et à les rapprocher $\mathrm{du}$ fonctionnement général de l'école, ouverte de 8 heures à 22 heures. Trois types d'accès ont ainsi été mis en place : heures d'ouverture traditionnelles (10h3017h30), avec présidence de salle et magasinier pour la communication des documents; heures de travail en « autodiscipline » (17h30-20h), sans la présence de personnel de la médiathèque, après adhésion à une charte de bonne conduite; heures de cours avec mise à disposition d'une personne du service pour la communication de documents des magasins et/ou l'assistance à la recherche (organisation possible entre $9 \mathrm{~h}$ et $20 \mathrm{~h}$ ). 
Pour autant, et malgré un taux de fréquentation élevé de la salle de lecture, peu d'interprètes (élèves et professeurs) fréquentent aujourd'hui les lieux. Même si la difficulté à trouver rapidement une partition peut être vécue comme un échec par de jeunes musiciens qui évoluent depuis l'enfance dans un système très sélectif où la compétition quotidienne rend difficile à vivre le fait de " ne pas savoir ", la question de l'accès aux sources ${ }^{6}$ n'explique pas tout.

D'autres éléments pèsent certainement autant : le poids du face à face du cours d'instrument et la parole du professeur qui privilégie souvent le travail de l'instrument à toute autre activité, la pression, le manque de temps et peut-être parfois la peur du savoir ${ }^{7}$ font sans doute partie des multiples raisons qui éloignent les élèves de cette ressource qui, depuis plus de deux siècles, leur est offerte.

Pour ne pas en rester là, professeurs et bibliothécaires ont mis en en place diverses actions. L'ensemble du personnel de la médiathèque (15 agents) s'est investi dans des activités nombreuses et régulières de présentation des collections, d'assistance et de formation à la recherche de répertoires. Cette envie de partager la connaissance des collections, conjuguée aux projets artistiques de certains élèves, a permis de faire émerger des sources singulières, originales et peu connues, souvent en lien avec l'histoire du Conservatoire et de ses traditions d'interprétation musicale. Du fait de la nature patrimoniale d'une grande partie du fonds, il a fallu accepter d'être très fréquemment confronté au dilemme conservation/communication. Un service de numérisation à la demande a été mis en place, mais le choix de communiquer des documents fragiles et difficiles à lire sur des supports de substitution (écrans, tablettes) a été fait pour permettre aux élèves de restituer des partitions oubliées ou passées de mode. Il n'a pas toujours été facile de gérer ce facteur de risque et la question fait régulièrement débat lors des réunions de service. Le bénéfice pédagogique et artistique ${ }^{8}$ ainsi que l'absence - à ce jour - de dégradations de documents incite cependant à poursuivre en ce sens.

Mais ce qui semble le plus à même de modifier le rapport des élèves avec le lieu et ses ressources vient de l'enseignement. Le fait que certains professeurs aient choisi de donner l'ensemble de leurs cours de méthodologie à la médiathèque semble annoncer, si ce n'est une petite révolution culturelle au sein de la communauté des élèves, du moins une évolution profonde. La nouvelle configuration de la salle de lecture n'est pas seule à l'origine de ce choix. Les relations sincères et amicales nouées depuis longtemps entre professeurs, bibliothécaires et magasiniers ont largement facilité la mise en place de ce qui était initialement une expérience avant de devenir une évidence.

\section{Le cours de " méthodologie de la recherche " à la Médiathèque Hector Berlioz, incubateur pour de nouveaux usagers}

La « méthodologie de la recherche " est un cours général qui s'adresse à l'ensemble des élèves en Master $1 \mathrm{du}$ Conservatoire, hormis les danseurs, les ingénieurs du son et les musicologues qui ont des cours spécifiques à leur spécialité. Par groupes de douze, la classe rassemble aussi bien des élèves en chant que dans les disciplines instrumentales classiques et contemporaines, en jazz, en musique ancienne, en écriture, en composition et en direction d'orchestre. À raison de trois heures bimensuelles ${ }^{9}$, il s'agit de l'un des rares cours où les élèves sont rassemblés, quelle que soit leur spécialité, et où les préoccupations des uns peuvent nourrir celles des autres. Les séances sont pensées comme un collège de discussion autour de la musique, de ses objets et de ses pratiques. Elles se déroulent partiellement ou intégralement à la Médiathèque Hector Berlioz, selon le choix du professeur.

L'objectif institutionnel du cours est d'« élaborer un projet de recherche préfigurant le Travail d'étude personnel (TEP) ${ }^{10}$ ", ce qui passe par la construction d'un objet de recherche, la collecte de ressources documentaires et la formulation d'une problématique. En Master 2, les élèves réalisent le travail de recherche (TEP) avec un tuteur spécialisé du sujet qu'ils ont choisi. L'objectif caché qui est fixé est de donner le goût de la recherche à un public peu habitué à fréquenter la salle de lecture de la médiathèque. Ces nouveaux usagers du lieu se l'approprient peu à peu pendant le cours de méthodologie, ils apprennent à se servir de ses outils pour devenir des lecteurs réguliers hors du cours.

Tant l'élaboration du projet de recherche que l'objectif caché du cours de méthodologie donnent lieu 
à trois types de lecteurs ou trois types d'utilisation de l'« espace médiathèque " : le lecteur qui vient pour le plaisir de la découverte, celui qui souhaite mettre en application le dialogue entre sa recherche et sa pratique, enfin celui qui produit du savoir par la formulation d'une pensée. Ces trois types d'utilisation correspondent au déroulement chronologique du cours. À raison de douze séances de trois heures ${ }^{11}$, chaque partie couvre quatre séances. Voici une mise en scène de la situation d'un élève qui suit le semestre de méthodologie et la manière dont son usage de la médiathèque évolue progressivement ${ }^{12}$.

\section{Le plaisir de la découverte}

Le premier groupe de séances a pour dessein, simple mais néanmoins socle du succès du cours, de transmettre aux élèves le plaisir de la découverte : d'un lieu, de son confort, de sa convivialité, de son accessibilité, de ses richesses, de ses outils et de ses possibilités d'utilisation. L'élève fréquente la salle de lecture et se promène à la rencontre des usuels. Il choisit, au gré de ses envies, des ouvrages de différents types - dictionnaires, revues, éditions monumentales, catalogues thématiques, biographies - qu'il synthétise, qu'il compare. On le retrouve, en dehors du cours, en train de lire des revues, confortablement installé sur les fauteuils de lecture. Entre deux répétitions d'orchestre, il trouve un lieu qui lui offre calme et confort, un refuge intellectuel sans exigence de résultat où il peut simplement se délecter d'une lecture... pour le plaisir. L'élève comprend rapidement que les usuels ne sont que la face cachée de l'iceberg, puisque les bibliothécaires l'emmènent dans les sous-sols pour lui faire découvrir la richesse du fonds : collections, ouvrages anciens, pièces uniques. Le musicien est extrêmement sensible à l'objet et à sa valeur puisqu'il a lui-même un instrument qui est la chose la plus précieuse qu'il possède. Être en face d'un objet précieux qui fait la musique - la partition, l'enregistrement, le manuscrit, la méthode provoque une émotion similaire à ce qu'il a l'habitude de recevoir lorsqu'il essaie un instrument nouveau. En dehors du cours, l'élève commence à se poser la question de son propre objet, de sa propre partition, réévalue les ressources en ligne qu'il fréquente déjà. Il n'hésite pas à prendre du temps, devant le bureau d'accueil, pour trouver le manuscrit de son concerto préféré, ou à défaut, son fac-similé.

Fort de cette découverte, l'élève apprend à se servir du catalogue pour trouver la pièce unique, déjà disponible dans le fonds et qui va le fasciner. Il commence par ailleurs à construire et sélectionner son environnement intellectuel face à l'infini des ressources auxquelles il a accès. Il sent alors que l'environnement humain du lieu - l'enseignant, les bibliothécaires, les autres élèves - est une aide précieuse. La médiathèque est un lieu qui lui permet de découvrir sans limite, il entre dans une boulimie de nouveauté : trouver de nouvelles œuvres, de nouvelles manières de jouer, de nouvelles techniques, de nouveaux outils, de nouvelles compétences.

\section{Recherche - pratique / Pratique - recherche}

L'élève a conscience que la recherche pourra lui faire tirer son épingle du jeu dans un monde de la musique tellement sélectif, mais il faut pour cela qu'elle soit directement reliée à son activité de musicien. Comment interroger sa pratique ? C'est le propre de la deuxième section du cours de méthodologie : mettre en place un dialogue, un va-et-vient permanent entre la recherche et la pratique par la mise en application des découvertes.

Derrière l'espace « cours » de la médiathèque, le musicien a accès à quatre studios de travail dont l'un est équipé d'un piano, les autres de pupitres et de matériels d'écoute en multicanal pour certains. Le studio de travail est un espace que le musicien connaît bien, puisqu'il sait s'enfermer pendant des heures pour pratiquer son instrument et réaliser un exercice jusqu'à la perfection. Il maîtrise parfaitement cet espace mais ce qui va changer dans sa démarche, c'est de mettre en application et faire fleurir des découvertes personnelles et non pas ce qu'il a reçu d'un professeur. L'élève peut écouter de la musique, en déchiffrer sur son instrument, essayer des techniques de jeu, des doigtés, des positions, des modes de production. En outre, habitué à passer des heures à la recherche de son matériel idéal (cordes, anches, embouchures), il reproduit cette démarche face à des objets nouveaux et insoupçonnés. La recherche nourrit définitivement sa pratique. 

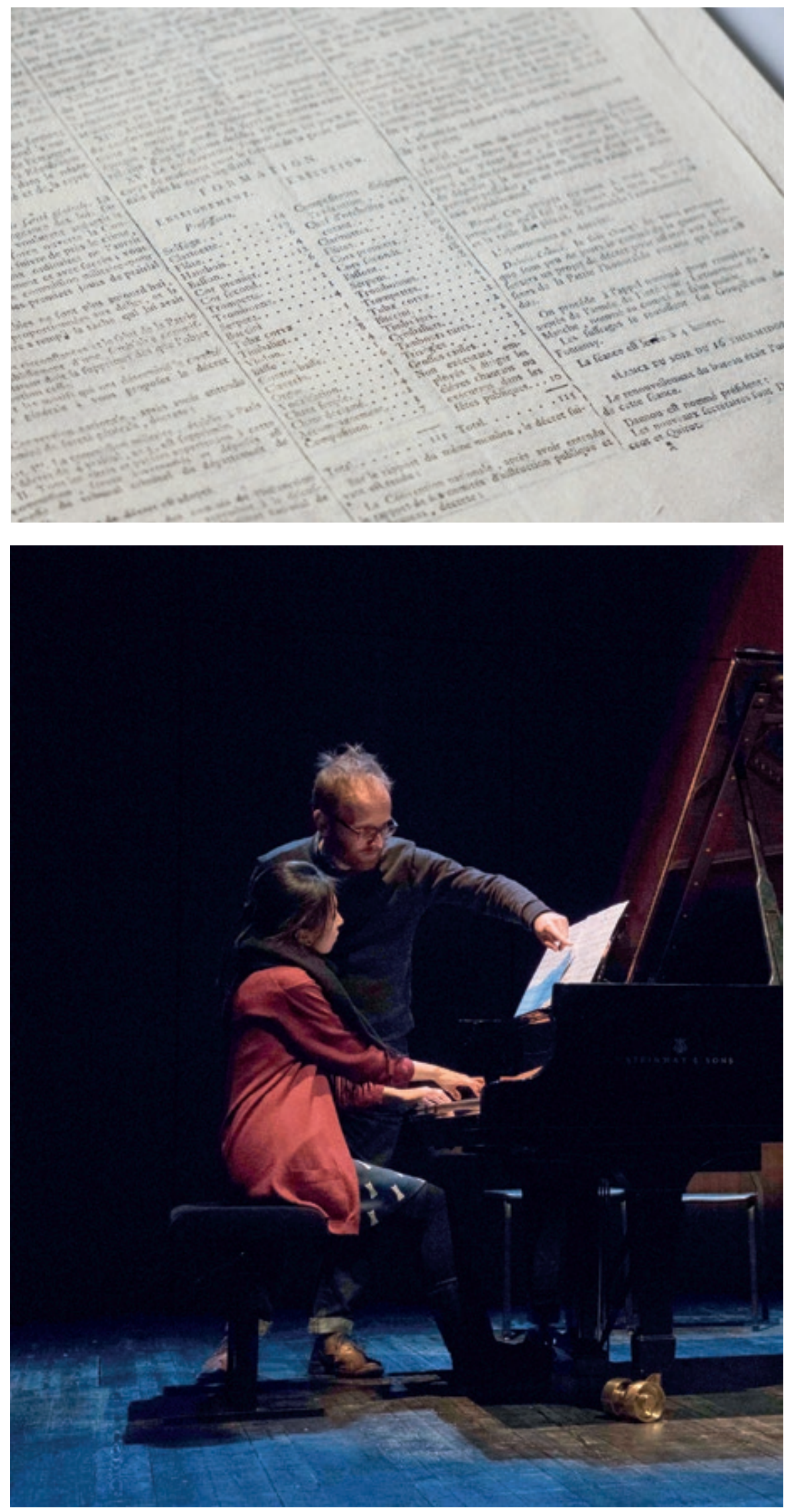

(Haut) Un extrait de la Gazette nationale du 21 thermidor an 3 (coll. Médiathèque Hector Berlioz) (Bas) Le face-à-face traditionnel élève-professeur : un cours de piano 

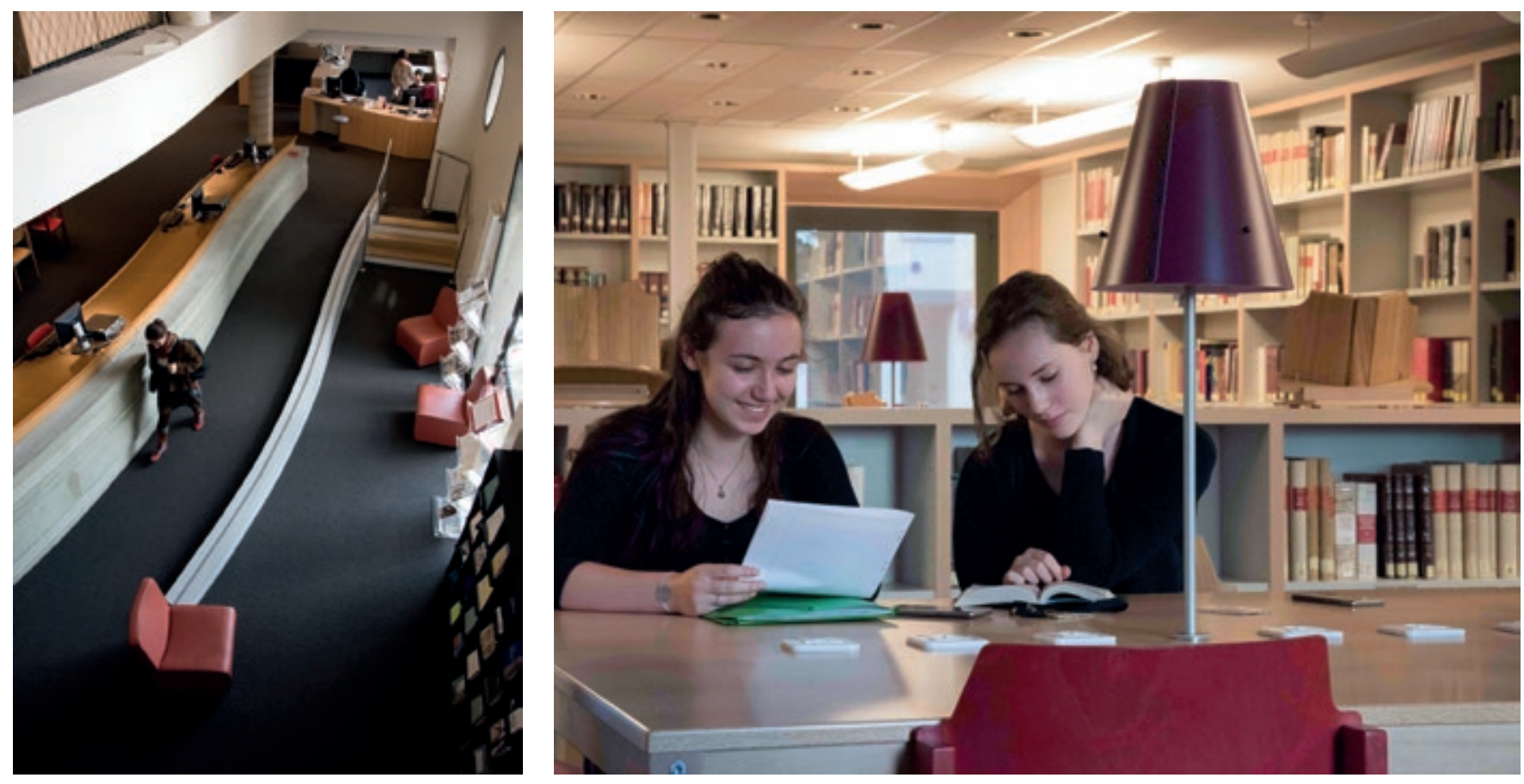

Travail à la médiathèque

Peu à peu, l'élève inscrit ce phénomène de recherche au cœur de son activité quotidienne. S'il est en période de préparation de concours internationaux, sa recherche va concerner l'optimisation du temps de travail ou la manière d'utiliser son corps. S'il est en passe de devenir enseignant, sa recherche va concerner les méthodes, les exercices reliés au répertoire. S'il joue d'un instrument rare, il va rechercher du répertoire ; s'il compose, il va chercher à établir des filiations stylistiques, à déceler un acte de création.

Ces quelques exemples montrent que la pratique nourrit définitivement la recherche. Peu de musiciens en ont conscience, et pourtant leur savoirfaire, qu'ils dédient principalement à la scène, est unique pour la recherche en musique. Tout comme un danseur, un musicien, qu'il soit interprète, compositeur, improvisateur ou musicologue, est par nature un chercheur. Le questionnement est permanent pour lui, et qu'il défende une partition existante ou qu'il en crée de nouvelles, il tente de s'exprimer de la façon la plus personnelle possible en tentant de transmettre à l'auditeur ses partis pris esthétiques les plus intimes ${ }^{13}$.

À l'instar de la revue en ligne du conservatoire, le lieu "médiathèque " est intégralement pensé pour le croisement entre recherche et pratique. Le cours de méthodologie doit donc faire prendre conscience au musicien que ce lieu lui est destiné et que la compétence qu'il apporte à la recherche en musique est considérable et peut/doit produire du savoir, au-delà de l'expérimentation personnelle. Quelquefois, l'étape de la formulation est nécessaire, et ce sont précisément des textes consacrés à l'aller-retour entre pratique et recherche qui sont l'objet de cette revue en ligne, dont le contenu est proposé par les professeurs du Conservatoire $^{14}$.

La troisième section du cours de méthodologie est consacrée à la formulation, étape qu'il faut passer habilement et sereinement pour ne pas abandonner définitivement son travail de chercheur-musicien. 


\section{Formuler une pensée}

Bien qu'aimant le lieu " médiathèque ", le musicien se prête au jeu de la rédaction avec plus ou moins d'aisance, de plaisir. Il a un instinct de chercheur et passe un temps infini à comprendre quelle est la meilleure réalisation d'un savoir-faire technique ; cette recherche est intuitive et ne nécessite pas d'être formulée. Toutefois, elle peut produire une compétence inédite extrêmement productive. Le professeur de méthodologie a pour mission d'accompagner l'élève, main dans la main, du studio de travail à la table en un aller-retour permanent.

L'élève a la curiosité nécessaire mais il a la crainte soit de ne pas savoir, soit de devoir remettre en question ce qu'il a toujours su. Le professeur de méthodologie et les bibliothécaires doivent le rassurer et lui apprendre à douter. Habitué aux cours d'instrument en tête à tête, l'élève a besoin d'un soutien individuel, d'un guide personnalisé. C'est le propre du tuteur de Master 2, mais le professeur de méthodologie peut se placer dans la position du passeur et mettre en place des stratégies pédagogiques pour donner cet aspect individualisé à son enseignement lors de la dernière étape du cours.

Finalement, l'élève réalise un Travail d'étude personnel, écrit en Master 2, qui peut prendre des formes très différentes : mémoire, préface d'édition, livret de CD ou de DVD, notes de programme, article de presse, journal de bord d'une expérience artistique, dossier de présentation d'un projet artistique, dissertation, support multimédia. La médiathèque l'incite à donner vie à son projet par un concert ou une présentation en salle de lecture. De chaque promotion d'une centaine d'élèves ressort une poignée de nouveaux usagers qui deviennent des lecteurs réguliers, habitués au lieu, et qui le fréquentent encore bien plus souvent après la réalisation de leur projet de Master.

Le propre de l'enseignement est de guider l'élève vers son autonomie. Le cours de méthodologie donne les outils pour être autonome face à la recherche. Autonome n'est toutefois pas synonyme de solitaire puisque l'élève est engagé dans des projets collectifs, la discussion autour des sources, la formulation de questions. L'organisation et l'atmosphère studieuse et humaine qui règnent à la Médiathèque Hector Berlioz en font un lieu unique, tant pour le chercheur-musicien expérimenté que pour le musicien-chercheur qui fait ses premières armes en cours de méthodologie, à l'âge de 16 ou 25 ans.

$\mathrm{Du}$ point de vue de la bibliothèque, le premier bilan que l'on peut faire de cette expérience d'enseignement in situ incite à envisager l'avenir avec optimisme et enthousiasme. Ce cours a généré beaucoup d'activité et de lien social dans l'espace de consultation : des recherches et des découvertes de répertoires ont été partagées par des musiciens qui ne se seraient pas rencontrés ailleurs. Il a également conduit - et ce n'est pas là son moindre mérite - bibliothécaires et magasiniers a construire un nouveau rapport avec les collections musicales, plus direct, plus proche et surtout plus sensible. Ils seraient les premiers déçus si l'expérience prenait fin : un peu comme si leur travail pouvait perdre de son sens.

\section{NOTES}

1- Loi portant établissement d'un Conservatoire de musique à Paris pour l'enseignement de cet art. Voir Pierre, C., Le Conservatoire national de musique, Paris, Imprimerie nationale, 1900, p. 124.

2- Discours inaugural de B. Sarrette, premier directeur du Conservatoire. Voir Pierre, C., B. Sarrette et les origines du Conservatoire national de musique et de déclamation, Paris, Delalain, 1895, p. 187.

3- Les premières sont composées de confiscations révolutionnaires, de collections de Versailles et de musiques révolutionnaires.

4- Nouveau nom de la bibliothèque du Conservatoire.

5- 133000 partitions, 23400 livres et plus de 45000 enregistrements sonores au $1^{\text {er }}$ janvier 2018.

6- La majorité des collections est conservée en magasins et le catalogue semble plus adapté à un usage professionnel que grand public.

7- Il n'est pas rare qu'un élève évoque le risque de moins bien jouer lorsque l'on « réfléchit ».

8- En quatre ans, plus d'une vingtaine de concerts sur des répertoires uniques issus des fonds de la médiathèque ont été donnés, sur place ou dans des salles de concert.

9- Ou une heure trente hebdomadaire.

10- Présentation de la discipline sur le site internet du Conservatoire de Paris [en ligne] : http://www.conservatoiredeparis.fr/disciplines/lesdisciplines/les-disciplines-detail/discipline/methodologie-de-recherche/ (consulté le 28/02/2018).

11- Ou de 24 séances d'lh30.

12- Le présent témoignage reprend de nombreuses réflexions menées conjointement avec Yves Balmer, professeur de méthodologie, et Patricia Frechon, bibliothécaire. Il met davantage en scène la figure de l'interprète que celle du compositeur.

13- Voir Bruno Mantovani, directeur du Conservatoire, dans la présentation de «La revue du Conservatoire », sur le site internet du Conservatoire de Paris [en ligne] : http://larevue.conservatoiredeparis.fr/ (consulté le 28/02/2018).

14- Ibid. 